Revue d'histoire de l'Amérique française

REVUE D.HISTOIRE DE L'AMÉRIQUE FRANÇAISE

FLEMING, Keith R., Power at Cost. Ontario Hydro and Rural Electrification, 1911-1958. Montréal et Kingston, McGill-Queen's University Press, 1992. 326 p. 39,95 \$

\title{
Claude Bellavance
}

Volume 47, numéro 2, automne 1993

URI : https://id.erudit.org/iderudit/305225ar

DOI : https://doi.org/10.7202/305225ar

Aller au sommaire du numéro

Éditeur(s)

Institut d'histoire de l'Amérique française

ISSN

0035-2357 (imprimé)

1492-1383 (numérique)

Découvrir la revue

Citer ce compte rendu

Bellavance, C. (1993). Compte rendu de [FLEMING, Keith R., Power at Cost. Ontario Hydro and Rural Electrification, 1911-1958. Montréal et Kingston, McGill-Queen's University Press, 1992. 326 p. 39,95 \$]. Revue d'histoire de l'Amérique française, 47(2), 273-276. https://doi.org/10.7202/305225ar d'utilisation que vous pouvez consulter en ligne.

https://apropos.erudit.org/fr/usagers/politique-dutilisation/ 
FLEMING, Keith R., Power at Cost. Ontario Hydro and Rural Electrification, 1911-1958. Montréal et Kingston, McGill-Queen's University Press, 1992. 326 p. 39,95\$

Quelques années à peine après sa création en 1906, Hydro-Ontario inscrivait l'approvisionnement des campagnes en énergie électrique parmi ses priorités. Ce faisant, l'entreprise d'État se démarquait de la plupart des compagnies d'électricité canadiennes et américaines, alors beaucoup plus enclines à exploiter les marchés lucratifs des grands centres. En fait, entre le début des années 1910 et la fin de la Seconde Guerre mondiale, l'Ontario a fait figure de pionnière en matière d'électrification rurale en Amérique du Nord. Desservir fermiers et habitants de petites localités rurales a posé des défis considérables aux ingénieurs et aux administrateurs d'Hydro-Ontario. Keith R. Fleming nous livre ici la première étude en profondeur du programme d'électrification rurale dans cette province.

L'ouvrage adopte un plan d'ensemble essentiellement chronologique. On s'est aussi inspirés de la relation des faits saillants de l'histoire d'HydroOntario dans ses rapports avec le milieu rural pour nommer la plupart des douze courts chapitres qui le composent («Laying the Foundations», «The Uniform Rate and Rural Bonuses», «The Battle for the Bruce», «Responses to the Depression», «The Five Year Plan», etc.). Quant au titre général de l'ouvrage, il nous renvoie à un problème fondamental pour toute entreprise de services publics - qu'elle soit société d'État ou compagnie privée celui de sa politique tarifaire. Vendre l'énergie au prix coûtant, tel a été en effet le mandat confié par le législateur à Hydro-Ontario, qui en a tôt fait un 
slogan publicitaire d'une rare efficacité. Le livre de Fleming le montre très bien: la réalité qui se cachait derrière l'expression power at cost était beaucoup plus complexe qu'il n'y paraissait au premier abord. Comment déterminer ce qu'est le prix coûtant lorsque la clientèle est à ce point diversifiée (industries, consommateurs urbains, commerces, fermiers, habitants de petites localités rurales, etc.)? Doit-on considérer le coût de production de l'énergie aux centrales seulement ou y inclure également les frais de transport et de distribution? La quantité d'énergie requise et le profil de consommation sont aussi déterminants sur le prix de revient. Ainsi, un abonné requérant une puissance élevée à certaines périodes du jour seulement est susceptible d'exercer une bien plus forte pression sur le réseau qu'un autre usager dont la consommation d'énergie serait plus échelonnée dans le temps. La répartition spatiale des clientèles est un autre facteur crucial en matière de tarification. Les coûts de distribution de l'électricité à une population rurale disséminée sur un vaste territoire sont en effet beaucoup plus élevés qu'en milieu urbain. Pour partager le plus rationnellement possible les frais d'exploitation du réseau, Hydro-Ontario a dû créer au fil des ans plusieurs classes d'usagers ainsi que des territoires administrativement distincts. Avant la fin de la Première Guerre mondiale cependant, l'électrification des campagnes en était encore à ses premiers balbutiements. Certes, quelque 132 petites localités étaient desservies par l'entreprise publique en 1919, mais seulement 300 fermes, par contre, disposaient du service de l'électricité. D'ores et déjà, les ingénieurs et dirigeants d'Hydro-Ontario avaient pris conscience de la nécessité de jumeler exploitations agricoles, hameaux, villages et petites villes s'ils voulaient augmenter la charge sur le réseau et ainsi optimiser les investissements. C'est pourquoi, ils créèrent, en 1920, les districts ruraux (Rural Power Districts), entités administratives regroupant divers types d'usagers au sein d'un territoire relativement homogène. Les coûts d'exploitation y étaient calculés pour l'ensemble du district, mais une grille tarifaire comportant huit classes d'usagers permettait d'ajuster les prix de manière à refléter la diversité de la clientèle. Une telle formule élargissait cependant considérablement le mandat d'Hydro-Ontario qui, créée pour répondre aux besoins des services municipaux de nombreuses villes ontariennes, n'avait pas eu accès jusqu'alors à la vente au détail. Désormais, un nombre grandissant d'abonnés allaient dépendre directement d'elle.

Mais l'instauration de districts ruraux ne faisait pas que conférer de nouveaux pouvoirs à la société d'État. Elle introduisait aussi une certaine péréquation en faveur des fermiers et autres habitants des campagnes ontariennes, ce qui allait directement à l'encontre de la politique tarifaire officielle de l'entreprise: celle de l'énergie au prix coûtant. L'auteur met en évidence à quel point de telles dispositions étaient essentielles au succès du programme d'électrification rurale. Sans elles, le prix de l'électricité aux agriculteurs aurait été tellement élevé que seuls les plus aisés d'entre eux auraient pu s'offrir ce «luxe». Réduire les tarifs permettait au contraire d'augmenter la demande et, par conséquent, de diminuer le coût de revient de l'énergie. Voilà pourquoi, à partir de 1921, l'État ontarien acceptait de participer au financement des nouvelles lignes de transport et de distribution en 
milieu rural. Tout le programme d'électrification rurale a d'ailleurs été marqué par cette obligation de soutenir un marché très fragile.

Au fil des ans, Hydro-Ontario a dû faire preuve de beaucoup de souplesse et d'imagination pour ne pas perdre l'appui de l'État ou s'aliéner une clientèle de plus en plus exigeante. Tantôt, c'était les élus de petites villes du sud de la province qui, misant sur l'électricité pour attirer des entreprises industrielles, dénonçaient les tarifs élevés qu'exigeait Hydro-Ontario (ce mouvement se transforma rapidement vers 1920 en une campagne pour l'instauration d'un tarif uniforme pour l'ensemble de la province). Tantôt ce furent les fermiers qui s'indignaient de ce que seuls les citadins aient accès au confort et aux nombreux autres avantages que conférait un service d'électricité fiable et abordable. Dominée par les grands centres, l'association des municipalités assurant le service de l'électricité s'objecta longtemps à ce qu'Hydro-Ontario prenne quelque distance par rapport au principe du power at cost et soutienne certaines catégories d'abonnés. Pour leur part, les gouvernements qui se sont succédé à Queen's Park ont le plus souvent su tirer profit de la situation, s'attachant par des mesures relativement peu coûteuses - et grâce à la complicité des dirigeants d'Hydro-Ontario — une bonne part du vote rural.

L'ouvrage de Keith R. Fleming recèle bon nombre d'informations précieuses sur les difficultés rencontrées par Hydro-Ontario dans la réalisation de son programme d'électrification des campagnes. Les chapitres portant sur la période 1930-1945 illustrent très bien, par exemple, comment la crise, puis la guerre, ont imposé des contraintes nouvelles à la compagnie d'électricité ontarienne qui réussit malgré tout à poursuivre l'extension de son réseau. Le chapitre six nous la montre, à la fin des années 1920, tentant de protéger un monopole de fait des marchés de l'électricité ontariens après l'incursion d'une entreprise américaine. Cet épisode passionnant, qui se termine brusquement avec le déclenchement de la grande crise des années 1930, est situé par Fleming dans le contexte plus général d'une véritable guerre idéologique à l'échelle nord-américaine, celle que se livraient alors Hydro-Ontario et la National Electric Light Association (NELA), un organisme dont la raison d'être était de promouvoir les intérêts des grands producteurs et distributeurs privés aux États-Unis et de faire obstacle au mouvement de municipalisation des services de l'électricité.

L'auteur aborde dans ce livre plusieurs thèmes centraux en histoire de l'électricité: la nécessité pour les entreprises productrices de promouvoir les usages de l'électricité afin de créer un marché, le rôle central de l'État aussi bien en matière de réglementation des distributeurs que de soutien à la modernisation de l'infrastructure de transport, l'impact des mouvements d'opinion, etc. Malgré l'intérêt de certaines analyses et la qualité de la narration, l'ouvrage adopte une approche somme toute assez traditionnelle qui tient plus de l'histoire politique que de l'histoire économique et sociale. Si l'auteur ne nous épargne guère de détails sur les enjeux de la dizaine d'élections provinciales qui furent déclenchées entre 1911 et 1960, sur les commentaires de la presse et sur les relations parfois tumultueuses entre Hydro-Ontario et tel groupe de citoyens, il est par contre beaucoup moins 
loquace sur des questions pourtant centrales comme la transformation du profil de consommation d'énergie des diverses catégories d'abonnés ruraux, l'emprise territoriale du réseau rural, l'évolution globale d'Hydro-Ontario, etc. De manière plus générale, on regrettera que Fleming n'ait pas tenté une analyse plus systématique de l'électrification rurale en Ontario. Les lecteurs intéressés aux dimensions économique, technologique ou spatiale de ce processus devront se contenter des quelques cartes présentées au début de l'ouvrage et des séries statistiques sommaires de l'appendice D sur l'étendue du réseau et sur le nombre d'agriculteurs et autres abonnés ruraux. C'est bien peu. Gageons qu'un jour ou l'autre une nouvelle étude viendra compléter le travail de Fleming qui exploitera de manière renouvelée la riche documentation conservée dans les archives d'Hydro-Ontario. En attendant, Power at Cost n'en représente pas moins, en dépit de ses lacunes, une contribution importante dans le domaine de l'histoire de l'électricité en Amérique du Nord.

Centre interuniversitaire d'études québécoises

Université du Québec à Trois-Rivières

CLAUDE BELLAVANCE 